\title{
Ultra-Low Temperatures in Ultra-High Magnetic Fields
}

Considerable effort continues to be invested in increasing the magnetic fields, both continuous and pulsed, available for condensedmatter research. But relatively little is being done to decrease the minimum temperatures obtainable in such fields. They are around $30-50 \mathrm{mK}$ for continuous fields and significantly higher for pulsed fields as the bore of the magnet is for technical reasons smaller. However, in many cases it is not the magnetic field $B$ but the ratio of $B$ to the temperature $T$ which is important in discovering and studying new physical states of matter.

While it seems impossible to increase today's $B / T$ ratio in continuous fields of 1000 $\mathrm{T} / \mathrm{K}$ by even a factor of two using state-ofthe-art magnet technology, we could gain a factor of 20 or more by decreasing the minimum temperature. One possibility is to combine a new type of dilution refrigerator based on a plastic heat exchanger with a nuclear demagnetization stage to reach a temperature of $1-2 \mathrm{mK}$ in the approximately $50 \mathrm{~mm}$ in diameter bore of state-of-the-art magnets generating continuous fields of $30 \mathrm{~T}$. We would then be in a position to extend frontiers in the study of intriguing materials such as ${ }^{3} \mathrm{He}$.

\section{Solid ${ }^{3} \mathrm{He}$}

Of all the solids, from a fundamental point of view ${ }^{3} \mathrm{He}$ is probably the most interesting. Owing to the lightness of the ${ }^{3} \mathrm{He}$ atom and its weak electronic interaction which hinders the formation of molecules, the amplitude of the zero-point vibration is very large (about $30 \%$ of the interatomic distance). The probability for an individual atom to tunnel from one site to a neighbouring site is therefore large (the tunnelling frequency is of the order of $1 \mathrm{mK}$ ). Solid ${ }^{3} \mathrm{He}$ can be easily obtained from the liquid phase by compression to pressures $P$ between $2.9 \mathrm{MPa}$ at 300 $\mathrm{mK}$ to $3.4 \mathrm{MPa}$ at $0 \mathrm{~K}$. It crystallises into a body-centred cubic (bcc) lattice at low pressures, and into a hexagonal close-packed

Giorgio Frossati has been Professor of Experimental Physics in the Kamerlingh Onnes Laboratory, University of Leiden, Postbus 9506, NL-2300 RA Leiden, since 1980. After studying physics at the University of São Paulo, Brazil, he joined the CRTBT, Grenoble, receiving his doctorate in 1978 . His interests are quantum fluids and solids, and cryogenics (his dilution refrigerator holds the world record of $1.9 \mathrm{mK}$ for continuous low temperatures).

Nei Oliveira Jr. has been Professor of Physics since 1972 in the Instituto de Física, University of São Paulo, Cx. Po. 20516, CEP 01498-970 São Paulo, Brazil, where he received his Ph.D. in 1966. He worked at the Francis Bitter National Lab, Cambridge, MA, USA, as postdoc and in 1977-79 as a staff member.

\author{
${ }^{1}$ G. Frossati, ${ }^{2}$ N.F. de Oliveira Jr. \\ ${ }_{1}^{1}$ Kamerlingh Onnes Laboratory, University of Leiden, The Netherlands \\ ${ }^{2}$ Instituto de Física, University of São Paulo, Brazil
}

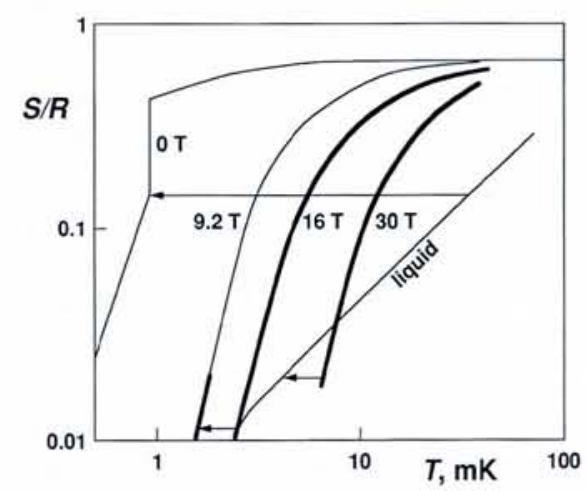

Fig. 1 - Entropy $S$ normalised with respect to the gas constant $R$ of solid ${ }^{3} \mathrm{He}$ at $9.2 \mathrm{~T}$ (measured; thin lines) and at $16 \mathrm{~T}$ and $30 \mathrm{~T}$ (calculated; thick lines). The solid entropy at zero field and the liquid entropy are also plotted. Several possible compression paths are indicated with arrows. At $16 \mathrm{~T}$, the entropy of the solid is estimated to equal that of the liquid at $T \approx 2.5 \mathrm{mK}$; at $30 \mathrm{~T}$, the entropies should intersect at 8-9 $\mathrm{mK}$.

lattice above about $10 \mathrm{MPa}$. Its compressibility of $\approx 5 \times 10^{-2} / \mathrm{MPa}$ is very large, and the additional localization of $3 \mathrm{He}$ atoms upon pressurisation strongly decreases the overlap of the atomic wave functions, so that the exchange frequency drops sharply.

It is expected that in this simple bcc system, the Heisenberg Hamiltonian would explain all experimental properties. However, the nuclear ordering temperature at the melting pressure has been found to be $1 \mathrm{mK}$ instead of $2 \mathrm{mK}$ [1] and the phase diagram is significantly more complex than that predicted by a simple Heisenberg Hamiltonian. It has a first-order region ending at a field $B_{\mathrm{c} 2}$ of about $0.5 \mathrm{~T}$ at $0 \mathrm{~K}$, where the spins are ordered in an up-up, downdown structure [2]. At higher fields, there is a second-order phase transition which increases in temperature up to $4 \mathrm{mK}$ and 10-12 T. It goes from paramagnetic to spinflop, decreasing for higher magnetic fields up to the critical field $B_{\mathrm{c} 2}[3]$ where full polarization is reached.

The explanation lies in the quantum nature of solid ${ }^{3} \mathrm{He}$. It has been shown that not only the exchange of two nearest-neighbour spins enters the Hamiltonian, but also threespin and two types of four-spin exchange (planar and folded), owing to the small size of the atom and its hard core. Large permutations are possible and minor contributions from five- and even six-spin exchange have been introduced.

Melting curve and entropy measurements (Fig. $1 ;$ [4]) are compatible with the $B_{\mathrm{c} 2}$ value of about $16 \mathrm{~T}$ calculated using meanfield theory, but experiments [5] measuring $\mathrm{d} P / \mathrm{d} B$ at $T<1 \mathrm{~K}$ up to $11 \mathrm{~T}$ indicate, on extrapolation, a substantially higher $B_{\mathrm{c} 2}$ of $21.7 \pm 1 \mathrm{~T}$.

Fig. 1 for the entropy of solid ${ }^{3} \mathrm{He}$ measured up to $9 \mathrm{~T}$, with extrapolation to 16 and $30 \mathrm{~T}$, shows that below $8 \mathrm{mK}$ at $30 \mathrm{~T}$, the entropy of the solid should fall below that of the liquid; this will give a maximum in the melting curve. At first glance, one should therefore measure $P(T)$ in fields from 15 to $30 \mathrm{~T}$ as this will provide a primary thermometer of great interest for low-temperature, high-field experiments. One should also determine the high-field magnetization of the solid to establish the value of $B_{\mathrm{c} 2}$, as this will give a rigorous test of the multiple-spin exchange Hamiltonian theory.

\section{Polarized Liquid ${ }^{3} \mathrm{He}$}

Owing to a small (nuclear) magnetic moment and its Pauli spin susceptibility, liquid ${ }^{3} \mathrm{He}$ can only be weakly spin polarized in the magnetic fields sustained at present in the laboratory (about $12 \%$ at $30 \mathrm{~T}$ ). However, since solid ${ }^{3} \mathrm{He}$ can be strongly polarized, Castaing and Nozières suggested that the liquid could be polarized by melting the solid in a short time compared to $t_{1}$, the nuclear relaxation time of the solid magnetization. The magnetization should be conserved in the liquid, hopefully long enough to do an experiment. Several groups have successfully exploited the idea to test some interesting theoretical predictions (divergence of viscosity and thermal conductivity, large increase of the superfluid transition temperature, etc.).

By growing polarized solid ${ }^{3} \mathrm{He}$ from the superfluid $A_{1}$ phase (where only spin-up pairs of ${ }^{3} \mathrm{He}$ are superfluid) and quickly melting it, polarized superfluid ${ }^{3} \mathrm{He}$ was produced for a few seconds with a polarization equivalent to an $18 \mathrm{~T}$ static field [6] and a transition temperature of $3.2 \mathrm{mK}$. The increase in $T_{c}(B)$ relative to $T_{c}(B)$ was smaller than that predicted on the basis of the so-called "nearly metamagnetic" model [7]. This result together with an increase in viscosity upon polarization, a recent experiment of the relaxation heat discussed by P. Monceau on page 94 , and our measurements on the viscosity increase upon pola- 